\title{
Effects of the coating in single-mode slab waveguide with varying curvature: a new approach to understanding bend loss
}

\author{
Céline Durniak and John D. Love \\ Optical Sciences Group, Research School of Physical Sciences \& Engineering, Australian National \\ University, Canberra ACT 0200, Australia
}

\begin{abstract}
We present analytical and numerical results that describe and quantify bend loss. Our approach is based on the coupling between fundamental and cladding modes with changing curvature in single-mode waveguides with finite cladding and absorbing coating.
\end{abstract}

\section{INTRODUCTION}

The next generation of devices for processing optically transmitted data based on optical fibre or planar waveguide technology needs to be more compact as light-processing systems become more miniaturized. Therefore, it has become essential to change the optical path within a very small area, which requires the use of bent fibres. However, curved fibres or waveguides are necessarily lossy. The total bend loss in a curved fibre or waveguide has traditionally been quantified in terms of transition loss, associated with an abrupt change in curvature which occurs when moving from the straight section to the curved section, and pure bend loss associated with radiation from a bend of constant curvature.

The loss from a bent fibre is essentially confined to the plane of the bend. Accordingly, the complexity of the simulation can be significantly reduced by reducing the dimension of the system: the effects of bending on propagation can be described by a bent slab waveguide.

In a previous paper [1] we developed a new approach to the physical description and quantification of bend loss in arbitrarily bent single-mode fibres, which was based on the coupling of the fundamental mode to cladding modes due solely to curvature change along the fibre. This criterion has an analogy with the corresponding criterion for the design of approximately adiabatic single-mode depressed-cladding and W-fibres tapers. We showed that there was an optimum bend design criterion that can reduce bend loss regardless of curvature. Here our model now takes into account the absorptive effects of the coating.

This paper is organized as follow. We begin in Section II by defining our model of the bent single-mode slab waveguide, and then we investigate the influence of a finite coating taking account of the evolution of modal effective indices and modal field profiles, and absorption of mode power as the curvature is varied. Finally, we show how our model integrates earlier explanations of bend loss and coating absorption into a more comprehensive description.

\section{MODEL}

We study the TE modes of a bent slab waveguide consisting of a homogeneous core, surrounded by a homogeneous finite cladding, which in turn is surrounded by a finite homogeneous coating beyond which is air.

The core and cladding are assumed to be non-absorbing. As we consider only a slightly absorbing coating medium, we can use a perturbation approach to determine the modal power attenuation. Hence we solve the eigenvalue equation in the weak guidance approximation and calculate the modal field profiles for the non-absorbing waveguide by using the same method as in [1] for the modes of the bent corecladding-air waveguide but with the field now sets to zero at the coating-air interface. The attenuation in the coating is taken into account when evaluating total modal power [2].

In this model, the source wavelength $\lambda$ is taken to be 1.55 $\mu \mathrm{m}$, the bent core has uniform index $\mathrm{n}_{\mathrm{co}}=1.4507$ and halfwidth $\rho_{\mathrm{co}}=1.6 \mu \mathrm{m}$ to be single-mode. The cladding half-width $\rho_{\mathrm{cl}}$ is equal to $35 \mu \mathrm{m}$ with uniform index $\mathrm{n}_{\mathrm{cl}}=1.444$ to ensure that it is single-mode with respect to the core and cladding indices. The coating has a half-width equal to $75 \mu \mathrm{m}$ and a complex refractive index $n_{j}=1.53+i 10^{-4}$. We take the real part of the refractive index of the coating to be higher than the cladding refractive index. This is the case with most commercially available single-mode fibres that have a primary coating made of an acrylate compound, with a real refractive index above 1.5. The real part of the refractive index of the coating relative to the cladding index determines whether the fundamental-mode field is confined within the cladding, and therefore the extent to which the field will penetrate into the coating. The absorption will attenuate any field propagating around a fibre bend because of the outward displacement of the modal field in the plane of the bend. The magnitude of the attenuation will also depend on the fraction of the field in the coating, as well as the value of the absorption coefficient.

\section{VARIATION OF THE CURVATURE}

\section{A. Evolution of the Effective Indices and of the Mode Field Profiles}

The evolution of the real part of the modal effective indices in the range $1.4435-1.4625$ is plotted in Fig. 1 as the bend radius is varied. The higher-value index plots shown here above the solid line corresponds to bound modes whose wave-like fields are confined in the coating and whose effective index values are above that of the core index.

The $\mathrm{TE}_{1}$ mode corresponding to the fundamental mode in the core and the TE2 mode corresponding to the first cladding mode swap their field roles at a bend radius $\mathrm{R}$ equal 
to $11.4 \mathrm{~mm}$ (solid and dashed lines in Fig. 1). The $\mathrm{TE}_{2}$ mode subsequently swaps this field with the $\mathrm{TE}_{3}$ mode at a smaller bend radius and so on.

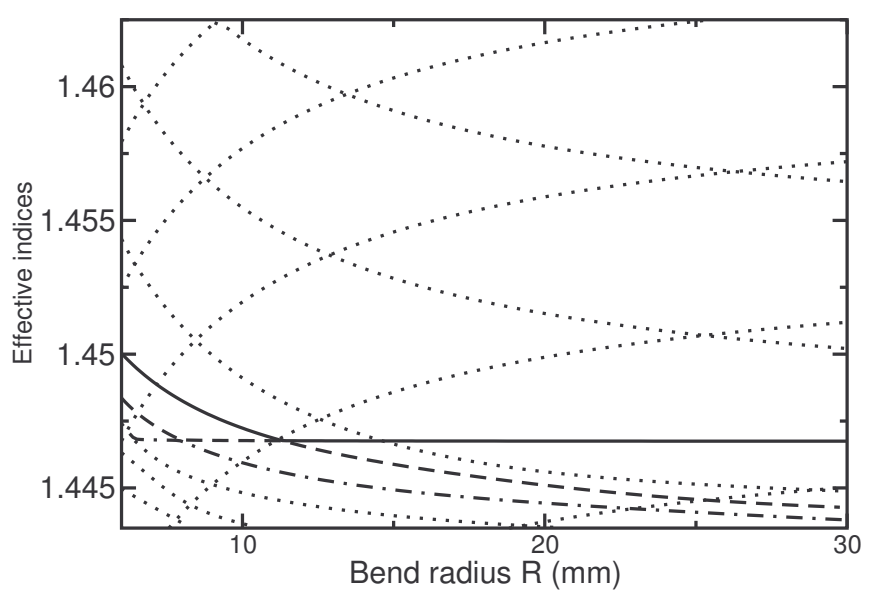

Fig. 1. Evolution of modal effective indices as a function of bend radius. The solid line corresponds to the $\mathrm{TE}_{1}$ mode, the dashed line to the $\mathrm{TE}_{2}$ mode and the dot-dashed line to the $\mathrm{TE}_{3}$ mode.

If we plot the evolution of the modal field for these modes for different bend radii, we have the evolution shown in Fig. 2. The modes are no longer confined entirely within the cladding and the fields extend into the coating region where they have oscillatory radial behaviour as would be expected. The approximate Gaussian shape of the fundamental mode of the unbent waveguide is associated with the $\mathrm{m}^{\text {th }}$ higher-order cladding mode of the bent waveguide whose order in turn depends on the bend radius. The Gaussian shaped mode is associated with the first cladding mode for $8<\mathrm{R}<11.4 \mathrm{~mm}$ [Fig. 2(b)] and with the fundamental mode for $\mathrm{R}>11.4 \mathrm{~mm}$ [Fig. 2(c)].

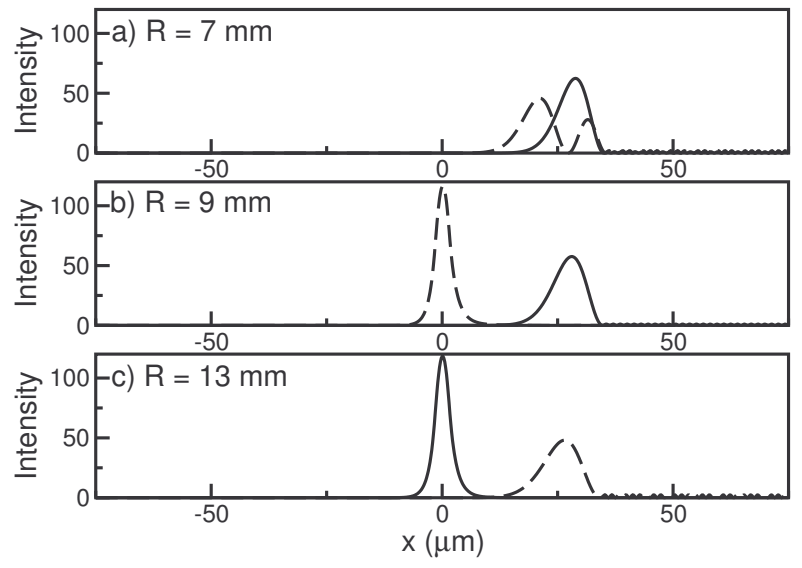

Fig. 2. Modal power distribution of a bent waveguide as a function of the transverse coordinate $\mathrm{x}$ for different bend radii. The styles of lines is the same as in Fig. 1 (solid line: $\mathrm{TE}_{1}$ mode and dashed line: $\mathrm{TE}_{2}$ mode). The modes' transverse electric fields have been normalized such that each mode carries a power equal to unity. The parameters values are given in Sect. II.
To calculate the modal attenuation due to absorption, we use a perturbation approach that first calculates the fraction of modal power propagating in the coating and then combines this with the imaginary part of the coating index [3].

In Fig. 3(a), the evolution of the power attenuation coefficient $\gamma_{\mathrm{i}}$ is plotted as a function of the bend radius where

$$
\gamma_{\mathrm{i}}=2 \mathrm{k}_{0} \frac{\operatorname{Re}\left(\mathrm{n}_{\mathrm{j}}\right) \operatorname{Im}\left(\mathrm{n}_{\mathrm{j}}\right)}{\mathrm{n}_{\mathrm{co}}} \frac{\int_{-\rho_{\mathrm{j}}}^{\rho_{\mathrm{cl}}} \psi_{\mathrm{i}}^{2} /(\mathrm{R}+\mathrm{x}) \mathrm{dx}+\int_{\rho_{\mathrm{clj}}}^{\rho_{\mathrm{j}}} \psi_{\mathrm{i}}^{2} /(\mathrm{R}+\mathrm{x}) \mathrm{d} \mathrm{x}}{\int_{-\rho_{\mathrm{j}}}^{\rho_{\mathrm{j}}} \psi_{\mathrm{i}}^{2} /(\mathrm{R}+\mathrm{x}) \mathrm{dx}}
$$

where $\mathrm{k}_{0}$ is the free space wave number, $\mathrm{R}$ is the bend radius, $\psi_{\mathrm{i}}$ corresponds to the solution of the wave equation for the $\mathrm{TE}_{\mathrm{i}}$ mode, $\mathrm{x}$ is the transverse coordinate, $\rho_{\mathrm{cl}}$ is the cladding radius, $\rho_{\mathrm{j}}$ is the position of the coating-air boundary, $\mathrm{n}_{\mathrm{co}}$ is the core refractive index and $\operatorname{Re}\left(\mathrm{n}_{\mathrm{j}}\right), \operatorname{Im}\left(\mathrm{n}_{\mathrm{j}}\right)$ are respectively the real part and imaginary part of the coating refractive index.

The solid, dashed and dot-dashed curves correspond to the $\mathrm{TE}_{1}, \mathrm{TE}_{2}$ and $\mathrm{TE}_{3}$ modes, respectively. For large bend radii, $\mathrm{R}>11.4 \mathrm{~mm}$, the $\mathrm{TE}_{1}$ mode has effectively zero attenuation as its field is confined predominantly within the core whereas the $\mathrm{TE}_{2}$ and $\mathrm{TE}_{3}$ mode fields are well spread across the core, cladding, and coating. For the second mode, the attenuation first decreases slowly with increasing bend radius for $\mathrm{R}<8$ $\mathrm{mm}$ as the mode is mainly in the cladding, then at $\mathrm{R}=8 \mathrm{~mm}$ it decreases abruptly as the mode moves into the core. At $\mathrm{R}=11.4 \mathrm{~mm}$ it abruptly increases and continues to increase when the mode moves back into the cladding, closer to the absorbing coating (see Fig. 2). Rapid jumps occur where the effective indices of pairs of modes are closest. The absorption of the coating modes is relatively large because their fields are confined predominantly within the coating.
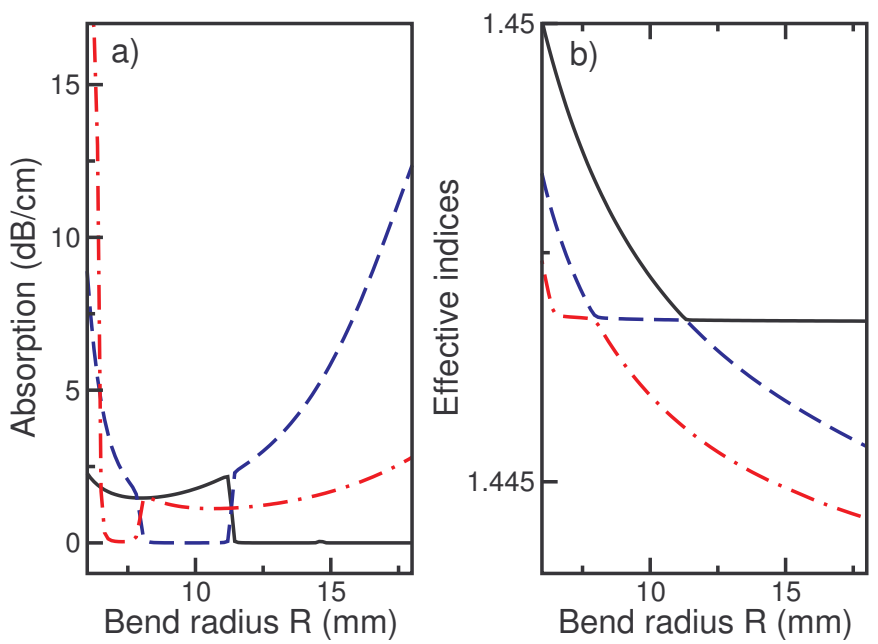

Fig. 3. a) Absorption and b) evolution of the effective indices for the first three modes as a function of the bend radius. The styles of lines are the same as in Fig. 1

\section{B. Absorption by The Coating}




\section{TOTAL BEND LOSS}

The simplest theoretical models considering pure bend loss were able to derive an approximate analytical formula for the attenuation of the fundamental mode relative to the axis of the bent fibre assuming an infinite cladding [3, 4]. This formula predicts that pure bend loss increases monotonically and approximately exponentially with decreasing bend radius.

Experimental measurements showed that for smaller bend radii pure bend loss exhibits a series of quasi-periodic maxima and minima superposed on a monotonic increase that were explained in terms of coupling between the fundamental mode and a whispering gallery mode propagating along the inside of the cladding-coating interface [5]. Alternative and more realistic models that take into account finite multicladding/coating layers and the loss effect of the coating via a reflection boundary condition at the cladding-coating interface have been shown to provide a plausible explanation for the bend loss oscillations [6-8].

Here we adopt a different approach. We suppose that the waveguide is bent along a circle of constant radius $\mathrm{R}$ and that it is illuminated by a Gaussian laser beam $\mathrm{E}_{\mathrm{s}}$ (with a waist of $3 \mu \mathrm{m})$. The attenuation of light power along the bent waveguide is due solely to absorption in the coating. The output power is given by

$$
\mathrm{P}(\mathrm{L})=\sum_{\mathrm{i}} \frac{\mathrm{n}_{\mathrm{co}}}{2} \sqrt{\frac{\varepsilon_{\mathrm{O}}}{\mu_{\mathrm{O}}}} \frac{\left|\int_{-\rho_{\mathrm{j}}}^{\rho_{\mathrm{j}}} \mathrm{E}_{\mathrm{s}} \psi_{\mathrm{i}} / \sqrt{\mathrm{R}+\mathrm{x}} \mathrm{dx}\right|^{2} \mathrm{e}^{-\gamma_{\mathrm{i}} \mathrm{L}}}{\int_{-\rho_{\mathrm{j}}}^{\rho_{\mathrm{j}}} \psi_{\mathrm{i}}^{2} /(\mathrm{R}+\mathrm{x}) \mathrm{dx}}
$$

where $\gamma_{\mathrm{i}}$ is given by (1), $\mathrm{R}$ is the bend radius, $\psi_{\mathrm{i}}$ corresponds to the solution of the wave equation for the $\mathrm{TE}_{\mathrm{i}}$ mode, $\mathrm{x}$ is the transverse coordinate, $\rho_{\mathrm{cl}}$ is the cladding radius, $\rho_{\mathrm{j}}$ is the coating radius, $\mathrm{n}_{\mathrm{co}}$ is the core refractive index $[2,3]$.

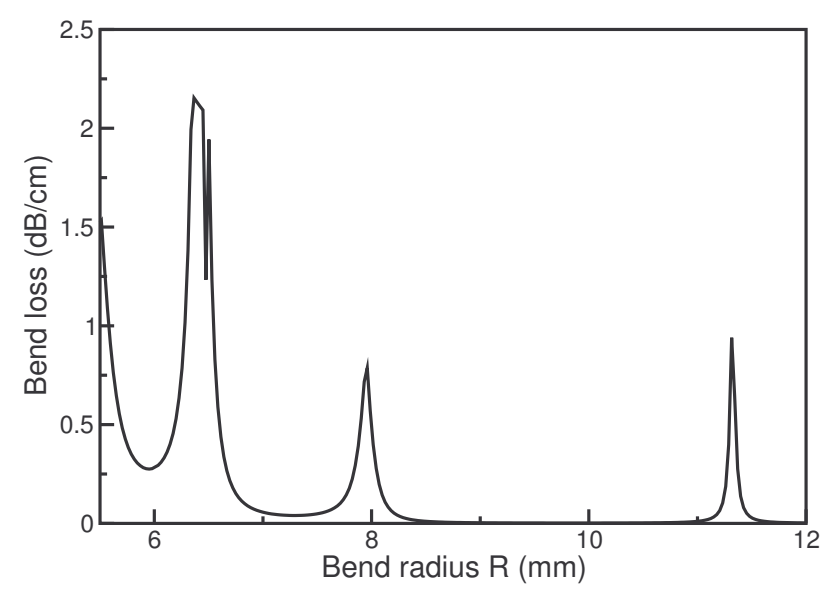

Fig. 4. Bend loss of a 3-metre long, uniformly bent waveguide as a function of the bend radius.
Using the procedure described above, we have determined the loss of the waveguide as a function of bend radius at 1.55 $\mu \mathrm{m}$. The results are shown in Fig. 4.

Peaks in bending losses of the coated single-mode waveguide occur where adjacent core and cladding modes swap field. For example, the peak at $\mathrm{R}=11.4 \mathrm{~mm}$ corresponds to a field swap between $\mathrm{TE}_{1}$ and $\mathrm{TE}_{2}$ modes (see Fig. 1). As the bend radius decreases, we observe field swaps between pairs of higher-order cladding modes.

\section{CONCLUSION}

In this paper, we have analysed the evolution of the effective indices and modal field profiles of a single-mode, finite-coating, finite-cladding bent waveguide of varying curvature along its length. We have considered the influence of the coating, which depends on both the real and imaginary parts of its refractive index in order to determine the fraction of the field absorbed in the coating. From this consideration we have developed an approach to describe bend loss, which shows that the local peaks in the loss occur at bend radii where modal field swapping occurs.

The present results are based on a waveguide model to help simplify the analytical and numerical analyses whilst retaining all the basic physical attributes of the problem.

\section{ACKNOWLEDGEMENT}

Dr Céline Durniak is supported by ARC Discovery grant DP0452918.

\section{REFERENCES}

[1] C. Durniak and J. Love, "Suppression of Cladding-Mode Coupling in Single-Mode Slab Waveguides of Varying Curvature", $31^{\text {st }}$ Australian Conference on Optical Fibre Technology \& Meeting of the Australian Optical Society, pp. 90-92, Melbourne 10-13 July 2006.

[2] J. A. Besley and J. D. Love, "Supermode analysis of fibre transmission," IEE Proc.-Optoelectron., vol. 144, no. 6, pp. 411-419, December 1997.

[3] A. W. Snyder and J. D. Love, Optical Waveguide Theory, Chapman and Hall, London 1983.

[4] D. Marcuse, "Bend Loss of Slab and Fiber Modes Computed with Diffraction Theory", IEEE J. Quant. Elect., vol. 29, no. 12, pp. 2957-2961, December 1993.

[5] A. J. Harris and P. F. Castle, "Bend Loss Measurements on High Numerical Aperture Single-Mode Fibers as a Function of Wavelength and Bend Radius", J. Lightw. Technol., vol. LT-4, no. 1, pp. 34-40, January 1986.

[6] I. Valiente and C. Vassallo, "New formalism for bending losses in coated single-mode optical fibres", Elect. Lett., vol. 25, no. 22, pp. 1544-1545, October 1989.

[7] L. Faustini and G. Martini, "Bend Loss in Single-Mode Fibers", J. Lightw. Technol., vol. 15, no. 4, pp. 671-679, April 1997.

[8] Q. Wang, G. Farrell, and T. Freir, "Theoretical and experimental investigations of macro-bend losses for standard single mode fibers", Opt. Exp., vol. 13, no. 12, pp. 4476-4484 June 2005. 